DOI: $\mathrm{xxx} / \mathrm{xxxx}$

\title{
Analysis of several subcycling schemes in partitioned simulations of strongly coupled fluid-structure interaction
}

\author{
Laurent De Moerloose*1,4 | Liesbeth Taelman ${ }^{2,4}$ | Patrick Segers ${ }^{2}$ | Jan Vierendeels Pa, $^{1,3}$ | Joris \\ Degroote $^{1,3}$
}

${ }^{1}$ Department of Flow, Heat and Combustion Mechanics, Faculty of Engineering and Architecture, Ghent University, Belgium

${ }^{2}$ IBiTech-bioMMeda, De Pintelaan 185, 9000 Ghent, Belgium

${ }^{3}$ Flanders Make, Belgium

${ }^{4}$ Both authors contributed equally to this work.

${ }^{5}$ Post mortem: the co-author has passed away on May 15th, 2018 while this article was under review.

\section{Correspondence}

* Laurent De Moerloose

Tel. +3292643297

Fax. +3292643590

Email: laurent.demoerloose@ugent.be

\section{Present Address}

Department of Flow, Heat and Combustion Mechanics, Sint-Pietersnieuwstraat 41-B4, 9000 Ghent, Belgium

\begin{abstract}
Summary
Fluid-structure interaction (FSI) simulations are used extensively to calculate the vibration of structures subjected to an internal or external flow. In the case of partitioned FSI simulations, a separate flow and structure solver are used, which requires some kind of coupling between both. The time step in both solvers is typically taken the same, but this unnecessarily leads to long calculation times when the time step is small due to stability reasons in one of the two solvers. Subcycling, the procedure where the time step of one solver is chosen smaller than the time step used in the other solver, may reduce the computational cost of the FSI simulation. The subcycling procedure can be either explicit or implicit, the latter implying the use of coupling iterations in each time step. Contrary to explicit subcycling, no stability analyses of implicit subcycling schemes are found in literature. In this paper, the numerical stability of the implicit subcycling procedure is investigated. The one-dimensional flow in an elastic cylindrical tube is studied analytically. The results of this analysis are subsequently compared to a partitioned two-dimensional axisymmetric FSI calculation with implicit coupling between the flow and structure solver.

\section{KEYWORDS:}

Implicit Fluid-Structure Interaction, Arbitrary Lagrangian Eulerian, Partial differential equations, Reynolds-Averaged Navier-Stokes, Stability
\end{abstract}

\section{1 | INTRODUCTION}

With the available modern-day computing power, fluid-structure interaction (FSI) simulations are increasingly used in academia to predict and understand the flow-induced vibrations in numerous applications. FSI simulations are particularly interesting for the study of movement-induced vibrations ${ }^{1}$, in which the movement of the structures reinforces the present flow instability. Nevertheless, FSI is also used to determine the fluid-elastic properties of structural vibrations with more limited amplitude, such as to avoid fretting in tube arrays subjected to axial flow ${ }^{11}$ or to quantify flutter dynamics in airplanes ${ }^{2]}$ or turbomachinery ${ }^{3}$. In classical FSI cases, the flow and structure solver apply the same time step in the transient simulations. Especially in the case of small amplitude structural motion, the maximal time step for which the structure solver remains accurate and stable might be significantly larger than the time step limit of the flow solver, which is imposed by requirements of turbulence modelling ${ }^{4}$, stability of time integration schemes ${ }^{5}$, the compressibility of the fluid ${ }^{6}$ and the presence of waves ${ }^{7}$. Since a coupling iteration happens at least once during every timestep, the required CPU time is large even though the structure barely moves during 
one time step. The FSI simulation can therefore be made more efficient by defining a smaller time step for the flow solver and a larger time step for the structure solver. Accordingly, multiple (smaller) time steps are performed in the flow solver before performing a coupling iteration and performing one (larger) time step in the structure solver, which is called subcycling. Even though uncommon, in some cases it can be beneficial to choose a structural time step which is smaller than the flow time step ${ }^{819}$.

Extensive research has been performed on subcycling in the field of aerodynamics ${ }^{10 \mid 1112}$, in which a significant reduction in simulation time and communication costs has been observed. Most authors perform stability analyses on explicit subcycling: partitioned simulations of fluid-structure interaction without coupling iterations between the flow simulation and the structural simulation in each time step. We consider a flow solver and a structure solver, respectively with time steps $\Delta t_{F}$ and $\Delta t_{S}$, such that a number $N_{S / F}$ is defined as: $N_{S / F}=\Delta t_{S} / \Delta t_{F}$. $N_{S / F}$ can be both larger than one (in which case $N_{S / F}$ is typically a positive integer) or smaller than one (in which case $N_{S / F}^{-1}$ is typically a positive integer). In case $N_{S / F}>1, N_{S / F}$ time steps $\Delta t_{F}$ of the flow are solved before one time step $\Delta t_{S}$ of the structure is calculated. The difficulty is to determine how the total fluid-structure interface displacement in a time step $\Delta t_{S}$ should be interpolated in the $N_{S / F}$ subcycles. Additionally, the question is what interface pressure should be transmitted to the structure solver at the end of the $N_{S / F}$ subcycles. In the conventional explicit subcycling procedure ${ }^{12}$, the interface pressure at the end of the last subcycling step is transmitted to the structure. The disadvantage is that the time step required to achieve a stable solution is limited and possibly lower than the time step limit imposed by the flow solver itself. In contrast, the stability of the subcycling method is preserved when two conditions are met. Firstly, the predicted displacement over one time step $\Delta t_{S}$ is evenly distributed among the $N_{S / F}$ subcycles. Secondly, the average interface pressure field computed during the $N_{S / F}$ subcycles is fed to the structure solver. These stability conditions have been validated for $2 \mathrm{D}$ inviscid calculations by Piperno ${ }^{11}$ and for 3D nonlinear problems by Farhat ${ }^{10}$, as well as for test cases outside the field of aerodynamics 13 .

Additionally, explicit subcycling is not limited to the coupling of finite volume and finite element methods. It has also been implemented successfully in the coupling of a lattice Boltzmann method and a discrete element method ${ }^{14}$ and is even used in structural dynamics 15116 . In the latter case, one assumes that the largest deformation of the structure is localized in one specific part of the domain, whereas the nodes outside of this region experience no or little motion. It would be a waste of computational resources to compute the entire domain with a small time step imposed by the strong yet local transient behaviour. Likewise, the global domain can be considered as an assembly of subdomains with their own time step size. This requires the existence of an interface across which these subdomains should be coupled, therefore requiring the use of an (explicit) subcycling method similar to FSI simulations. Analogously, subcycling has been implemented in fluid-fluid interactions ${ }^{[17}$ in order to model atmosphere-ocean interaction, where the time step limit is mainly imposed by the presence of fast gravitational waves $\frac{18}{\text {. }}$

Contrary to explicit subcycling, little research can be found about implicit subcycling. In traditional implicit FSI schemes, at least one coupling iteration between the flow solver and the structure solver is performed in every time step. Obviously, explicit subcycling schemes are easier to implement: no coupling iterations are required and thus all flow and structure variables are only calculated once. As such, explicit coupling is more flexible with respect to the use of different time steps in the solvers ${ }^{19}$. On the other hand, it is well-established that there are FSI problems for which the explicit coupling does not reach convergence, e.g. in high density fluids ${ }^{[20}$. In those cases, implicit coupling ${ }^{21}$ (or a monolithic solver ${ }^{22}$ ) is required to reach a stable solution, which is why more research into implicit subcycling is needed.

In this paper, an implicit subcycling scheme is proposed and its stability is investigated. Specifically, the temporal stability of the method is investigated, not the convergence stability of the coupling method. A monolithic analytic study on a onedimensional model for flow in an elastic tube is presented. The analytic results are subsequently verified by a numerical study in which the propagation of a sinusoidal flow wave in a straight tube is simulated using nonlinear two-dimensional axisymmetric partitioned implicit FSI simulations.

\section{2 | ANALYTIC STUDY}

\section{1 | Governing equations}

The one-dimensional flow in a straight, flexible tube shown schematically in Figure 1 is considered as test case as it allows analytic manipulations. Gravity and viscosity are not taken into account. The equations that describe the unsteady flow of the incompressible fluid are the continuity and the momentum equation, given by

$$
\frac{\partial s}{\partial t}+\frac{\partial s u}{\partial x}=0
$$




$$
\frac{\partial s u}{\partial t}+\frac{\partial s u^{2}}{\partial x}+\frac{1}{\rho_{f}}\left(\frac{\partial s \hat{p}}{\partial x}-\hat{p} \frac{\partial s}{\partial x}\right)=0
$$

in which $s$ represents the cross sectional area of the tube, $u$ the axial velocity, $x$ the axial coordinate, $t$ the time, $\rho_{f}$ the fluid density and $\hat{p}$ the pressure. In the remainder of this paper the kinematic pressure $p=\hat{p} / \rho_{f}$ will be used. In the structural subproblem, the circumferential and longitudinal deformation of the tube are neglected. The deformation in radial direction is determined by

$$
\rho_{s} h \frac{\partial^{2} r}{\partial t^{2}}+\chi \frac{\partial^{4} r}{\partial x^{4}}-\psi \frac{\partial^{2} r}{\partial x^{2}}+\eta\left(r-r_{0}\right)=\rho_{f}\left(p-p_{0}\right)
$$

with $r$ describing the inner radius, $\rho_{s}$ the wall density, $h$ the wall thickness and $p_{0}$ the pressure corresponding to the reference radius at rest ${ }^{[23} r_{0}$. This structural model is an extension to the so-called independent-rings model ${ }^{24}$, as the terms containing $\chi$ and $\psi$ take into account the longitudinal interaction between the tube segments. $\chi, \psi$ and $\eta$ are positive constants depending on the wall properties, where $\chi$ accounts for the inner action of the tube bending and $\psi$ for the shear deformation. $\psi$ is calculated as $\kappa G h$ with $\kappa$ the Timoshenko shear correction factor and $G$ the shear modulus. $\eta$ is given by $E h /\left(1-v^{2}\right) r_{0}^{2}$ with $E$ the Young's modulus and $v$ the Poisson's ratio.

The analytic study is performed with a monolithic approach in order to allow for a comprehensive study of the eigenvalues of the matrix relating the variables in subsequent time steps. As the temporal stability of the physical solution is investigated, it does not matter whether the solution of each time step is calculated with a partitioned or with a monolithic scheme. The conclusions drawn in the following analysis, are therefore also valid for a partitioned implicit FSI simulation. The fluid-structure interface condition is of the Dirichlet-Neumann type: the displacement of the structure is used as a boundary condition for the flow solver while the fluid pressure is transmitted to the structure solver.

\section{2 | Spatial discretization}

The tube with length $L$ is discretized in $N$ spatial intervals of equal length $\Delta x$, as shown in Figure 1 A first-order upwind scheme is used for the convective term in the momentum equation, while central discretization is used for the other terms in the flow equations. For the spatial discretization of the structural equation, the central difference scheme is applied. Subscripts $i$ and $i \pm 1 / 2$ indicate cell centers and faces, respectively.

To allow for analytic manipulation, the velocity, radius and pressure are subsequently linearized as the sum of a reference value (indicated with a subscript 0 ) and a perturbation term (indicated with a prime). Neglecting the terms which are quadratic in the perturbed quantities, this gives

$$
\begin{gathered}
\Delta x \frac{\partial 2 r_{0} r_{i}^{\prime}}{\partial t}+r_{0}^{2}\left(u_{i+1 / 2}^{\prime}-u_{i-1 / 2}^{\prime}\right)+2 r_{0} u_{0}\left(r_{i+1 / 2}^{\prime}-r_{i-1 / 2}^{\prime}\right)-\delta\left(p_{i+1}^{\prime}-2 p_{i}^{\prime}+p_{i-1}^{\prime}\right)=0 \\
\Delta x \frac{\partial\left(r_{0}^{2} u_{i}^{\prime}+2 r_{0} u_{0} r_{i}^{\prime}\right)}{\partial t}+r_{0}^{2} u_{0}\left(u_{i}^{\prime}-u_{i-1}^{\prime}+u_{i+1 / 2}^{\prime}-u_{i-1 / 2}^{\prime}+2 r_{0} u_{0}^{2}\left(r_{i+1 / 2}^{\prime}-r_{i-1 / 2}^{\prime}\right)+\frac{1}{2} r_{0}^{2}\left(p_{i+1}^{\prime}-p_{i-1}^{\prime}\right)=0\right.
\end{gathered}
$$

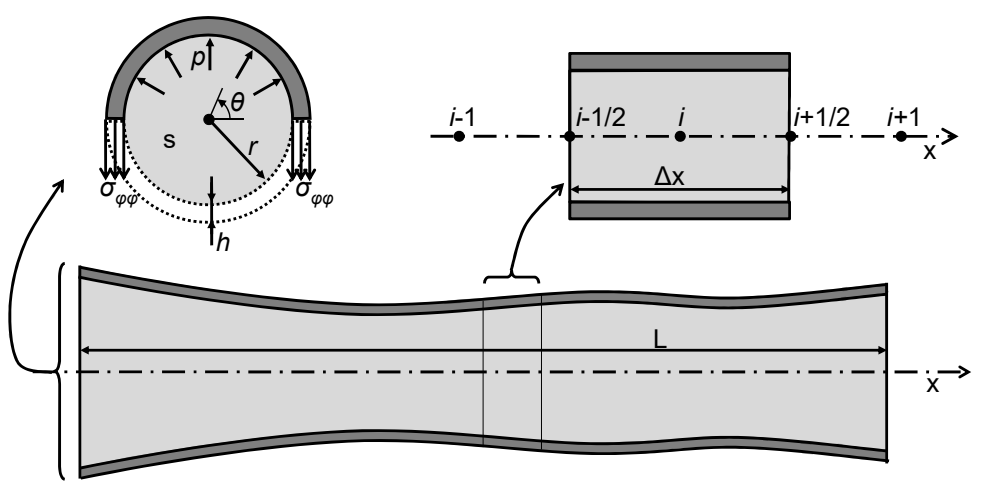

FIGURE 1 Schematic of the one-dimensional flow through a straight, elastic tube. 


$$
\rho_{s} h \frac{\partial^{2} r_{i}^{\prime}}{\partial t^{2}}+\frac{\chi}{\Delta x^{4}}\left(r_{i+2}^{\prime}-4 r_{i+1}^{\prime}+6 r_{i}^{\prime}-4 r_{i-1}^{\prime}+r_{i-2}^{\prime}\right)-\frac{\psi}{\Delta x^{2}}\left(r_{i+1}^{\prime}-2 r_{i}^{\prime}+r_{i-1}^{\prime}\right)+\eta\left(r_{i}^{\prime}\right)=\rho_{f}\left(p_{i}^{\prime}\right)
$$

for $u_{i} \geq 0$. As the pressure term is discretized with a central scheme, pressure stabilization is needed. Therefore, artificial diffusion is added in the continuity equation with coefficient ${ }^{25]} \delta=s_{0} / \pi /\left(u_{0}+\Delta x / \Delta t\right)$. This term disappears upon convergence of the solution.

\section{3 | Fourier equations}

The pressure, radius and velocity perturbation are then decomposed as the sum of $N$ Fourier modes. For the radius perturbation this results in

$$
r_{i}^{\prime}=\frac{1}{N} \sum_{l=0}^{N-1} \tilde{r}_{l} \exp \left(\jmath \omega_{l} i \Delta x\right)
$$

with $\omega_{l}=2 \pi l / L$ the angular wave number. As Equations (2) are linear in velocity, pressure and radius perturbation, the temporal stability of the flow in the flexible tube is determined by the temporal stability of each individual wave component. To study the different wave components separately, the governing equations with $r_{i}^{\prime}$ substituted with Equation (3) are projected on $\exp \left(j \omega_{\ell} i \Delta x\right)$ and similarly for $u_{i}^{\prime}$ and $p_{i}^{\prime}$. To keep the equations readable, the product $\omega_{\ell} \Delta x$ is substituted by $\theta_{\ell}$ and the tilde and subscripts are omitted.

$$
\begin{gathered}
\Delta x \frac{\partial 2 r_{0} r}{\partial t}+\jmath r_{0}^{2} \sin (\theta) u+2 \jmath r_{0} u_{0} \sin (\theta) r+2 \delta(1-\cos (\theta)) p=0 \\
\Delta x \frac{\partial\left(r_{0}^{2} u+2 r_{0} u_{0} r\right)}{\partial t}+(1-\exp (-\jmath \theta)+\jmath \sin (\theta)) r_{0}^{2} u_{0} u+2 \jmath u_{0}^{2} r_{0} \sin (\theta) r+\jmath r_{0}^{2} \sin (\theta) p=0 \\
\rho_{s} h \frac{\partial^{2} r}{\partial t^{2}}+\left(\frac{4 \chi}{\Delta x^{4}}(1-\cos (\theta))^{2}+\frac{2 \psi}{\Delta x^{2}}(1-\cos (\theta))+\eta\right) r=\rho_{f} p
\end{gathered}
$$

\section{4 $\mid$ Temporal discretization}

The time discretization is initally formulated assuming the time step size $\Delta t$ for both the flow and the structure. The backward Euler scheme (BE) is used for the time discretization of the flow equations in order to avoid older timesteps in the discrete equations. This yields

$$
\begin{gathered}
\frac{2 r_{0} \Delta x}{\Delta t}\left(r^{n+1}-r^{n}\right)+\jmath r_{0}^{2} \sin (\theta) u^{n+1}+2 \jmath r_{0} u_{0} \sin (\theta) r^{n+1}+2 \delta(1-\cos (\theta)) p^{n+1}=0 \\
\frac{r_{0}^{2} \Delta x}{\Delta t}\left(u^{n+1}-u^{n}\right)+\frac{2 r_{0} u_{0} \Delta x}{\Delta t}\left(r^{n+1}-r^{n}\right)+(1-\exp (-\jmath \theta)+\jmath \sin (\theta)) r_{0}^{2} u_{0} u^{n+1}+2 \jmath u_{0}^{2} r_{0} \sin (\theta) r^{n+1}+\jmath r_{0}^{2} \sin (\theta) p^{n+1}=0
\end{gathered}
$$

For the temporal discretization of the structure, either the BE scheme is used such that flow and structure are discretized in the same way or the operator defined by Hilber, Hughes and Taylor (HHT) ${ }^{26}$ is applied, which is more common for structures. The BE scheme for the structure yields

$$
\rho_{s} h \ddot{r}^{n+1}+\left(\frac{4 \chi}{\Delta x^{4}}(1-\cos (\theta))^{2}+\frac{2 \psi}{\Delta x^{2}}(1-\cos (\theta))+\eta\right) r^{n+1}=\rho_{f} p^{n+1}
$$

in which the acceleration and velocity are calculated as

$$
\ddot{r}^{n+1}=\frac{1}{\Delta t}\left(\dot{r}^{n+1}-\dot{r}^{n}\right) \quad \text { and } \quad \dot{r}^{n+1}=\frac{1}{\Delta t}\left(r^{n+1}-r^{n}\right) .
$$

An overdot signifies a time derivative.

For the HHT scheme, the internal and external forces in Equation (4c) are treated similarly and are replaced by a weighted average of the forces at the beginning and end of the time step, as opposed to a fully implicit treatment of the external forces 
in Hilber et al. ${ }^{26}$ This approach corresponds to the approach imposed in the structure solver, used for the numerical validation afterwards.

$$
\begin{aligned}
\rho_{s} h \ddot{r}^{n+1}+(1+\alpha)\left[\left(\frac{4 \chi}{\Delta x^{4}}(1-\cos (\theta))^{2}+\frac{2 \psi}{\Delta x^{2}}(1-\right.\right. & \left.\cos (\theta))+\eta) r^{n+1}-\rho_{f} p^{n+1}\right] \\
& -\alpha\left[\left(\frac{4 \chi}{\Delta x^{4}}(1-\cos (\theta))^{2}+\frac{2 \psi}{\Delta x^{2}}(1-\cos (\theta))+\eta\right) r^{n}-\rho_{f} p^{n}\right]=0
\end{aligned}
$$

The operator definition is completed by the Newmark formula ${ }^{27}$ for acceleration and velocity integration

$$
\begin{gathered}
\ddot{r}^{n+1}=\frac{1}{\beta \Delta t^{2}}\left(r^{n+1}-r^{n}\right)-\frac{1}{\beta \Delta t} \dot{r}^{n}-\left(\frac{1}{2 \beta}-1\right) \ddot{r}^{n} \\
\dot{r}^{n+1}=\dot{r}^{n}+\Delta t(1-\gamma) \ddot{r}^{n}+\Delta t \gamma \ddot{r}^{n+1}
\end{gathered}
$$

with $\alpha \in[-1 / 3,0]$ determining the numerical dissipation, $\beta=(1-\alpha)^{2} / 4$ and $\gamma=1 / 2-\alpha$.

\section{5 | Subcycling in the flow problem}

In the previous section, the same time step size $\Delta t$ was used for the time integration of the flow and the structure. For the analytic study of subcycling in the flow problem, the flow time step $\Delta t_{f}$ is chosen equal to half of the structural time step $\Delta t_{s}$, i.e. $N_{S / F}=2$. Consequently, $\Delta t_{s}=\Delta t$ is called 'a time step' and a flow sub-time step $\Delta t_{f}=\Delta t / 2$ is referred to as 'a subcycle'. Since two subcycles are solved in each time step, the displacement of the structure has to be interpolated in these subcycles. A jump or linear interpolation is not sufficient: due to the imposed Dirichlet condition in the flow solver, the calculated fluid pressure which is related to the interface acceleration, is the result of a double time derivative procedure. For the numerical stability as well as the physical accuracy of the solution, a continuous acceleration is desired in each node of the wall. Therefore, a third degree polynomial per point on the interface is used to prescribe the wall displacement during time step $n+1$. The four parameters in this polynomial are determined by the wall displacements $r^{n}$ and $r^{n+1}$ at the beginning and the end of the time step and by matching the discretized second derivative of the polynomial at the beginning and the end of the time step with the wall accelerations $\ddot{r}^{n}$ and $\ddot{r}^{n+1}$ as calculated by the structure solver. The wall displacement imposed during the first subcycle is then given by $r^{n+1 / 2}=\frac{r^{n+1}}{2}+\frac{r^{n}}{2}-\frac{3 i^{n+1} \Delta t^{2}}{16}+\frac{i^{n} \Delta t^{2}}{16}$ and $r^{n+1}$ during the second subcycle. Two new variables are introduced: $u^{n+1 / 2}$ and $p^{n+1 / 2}$, representing the fluid velocity and the pressure at the intermediate time $n+1 / 2$ between time step $n$ and $n+1$.

In case the BE scheme is used in both solvers, Equation (5) for the flow is reformulated for both sybcycles, which can be found in Appendix A The structure problem is completely described with Equation (6). In order to study the temporal stability, the system of equations is written in matrix form

$$
\begin{gathered}
A y=B z \\
\text { with } y=\left[r^{n+1} \dot{r}^{n+1} \ddot{r}^{n+1} r^{n+1 / 2} u^{n+1 / 2} u^{n+1} p^{n+1 / 2} p^{n+1}\right]^{T} \\
\text { and } z=\left[r^{n} \dot{r}^{n} \ddot{r}^{n} r^{n-1 / 2} u^{n-1 / 2} u^{n} p^{n-1 / 2} p^{n}\right]^{T}
\end{gathered}
$$

In case the BE scheme and the HHT scheme are used in the flow and the structural solver, respectively, the flow is also described by the same equations in Appendix A but the structure is instead governed by Equation (7).

The variables $r^{n-1 / 2}, u^{n-1 / 2}$ and $p^{n-1 / 2}$ do not occur in any equation, but are added to obtain a square amplification matrix $A^{-1} B$. The corresponding columns in the matrix B are zero-columns. The parameters shown in Table 1 are used to calculate the eigenvalues $\lambda_{i}$ (with $i=1, \ldots, 8$ ) of the amplification matrix $A^{-1} B$. The grid size and time step size are chosen sufficiently small $\left(\Delta x=10^{-4} \mathrm{~m}, \Delta t=10^{-5} \mathrm{~s}\right)$, so that the eigenvalues are unaffected by numerical dissipation in the considered wave number region. For both combinations of time integration schemes, two eigenvalues $\lambda_{1,2}$ of the amplification matrix $A^{-1} B$ are equal to one. Their eigenvectors correspond to the solution of the one-dimensional case. The stability of the FSI problem is thus determined by the absolute value of the other six eigenvalues.

BE-BE combination Using the BE scheme for the discretization of both the flow and the structural equations, the variable $p^{n}$ does not occur in the equations. Consequently, the eighth column of $\mathrm{B}$ is also zero in this particular case. Four eigenvalues are zero, corresponding to the spurious modes $e_{4}, e_{5}, e_{7}$ and $e_{8}$. A vector $e_{i}$ has the same length as $y$ and $z$ and contains a 1 in position $i$ and all other elements are 0 . These eigenvectors clearly result from the absence of the variables $r^{n-1 / 2}, u^{n-1 / 2}$, $p^{n-1 / 2}$ and $p^{n}$ or, equivalently, from the zero-columns present in $\mathrm{B}$. The amplitude of the other eigenvalues $\lambda_{7,8}$ is shown in Figure $2 \mathrm{a}$ as a function of the wave number $\omega$. Furthermore, performing a similar analysis on the system of Equations (5.6) 
TABLE 1 Dimensions of the model and material properties.

\begin{tabular}{crl|crl}
\hline $\mathrm{E}$ & 300000 & $\mathrm{~N} / \mathrm{m}^{2}$ & $v$ & 0.49 & \\
$\chi$ & 0 & $\mathrm{~kg} \mathrm{~m}{ }^{2} / \mathrm{s}^{2}$ & $\psi$ & 55 & $\mathrm{~kg} / \mathrm{s}^{2}$ \\
$\rho_{s}$ & 1200 & $\mathrm{~kg} / \mathrm{m}^{3}$ & $\rho_{f}$ & 1000 & $\mathrm{~kg} / \mathrm{m}^{3}$ \\
$u_{0}$ & 0.1 & $\mathrm{~m} / \mathrm{s}$ & $r_{0}$ & 0.005 & $\mathrm{~m}$ \\
$L$ & 0.05 & $\mathrm{~m}$ & $h$ & 0.001 & $\mathrm{~m}$ \\
\hline
\end{tabular}

without subcycling, yields only one relevant eigenvalue - the other eigenvalues are either equal to zero or one. This eigenvalue determines the temporal stability of the system without subcycling and is therefore also shown in Figure 2a. The use of subcycles has a clear negative effect on the damping behaviour for small wavenumbers $\omega$. The amplitude remains, however, smaller than one for all wavenumbers and thus a stable solution should be obtained.

BE-HHT combination In case the HHT operator is used for the structure combined with the BE scheme for the flow, three eigenvalues $\lambda_{3,4,5}$ equal to zero are found, corresponding to the eigenvectors $e_{4}, e_{5}$ and $e_{7}$. The amplitude of the three remaining eigenvalues $\lambda_{6,7,8}$ is shown in Figure $2 \mathrm{~b}$ According to this figure, the use of subcycling reduces the amplitude of the eigenvalues of the BE-HHT scheme and the FSI problem remains stable for all wave numbers. Other values of $N_{S / F}$ are not included in this analysis.
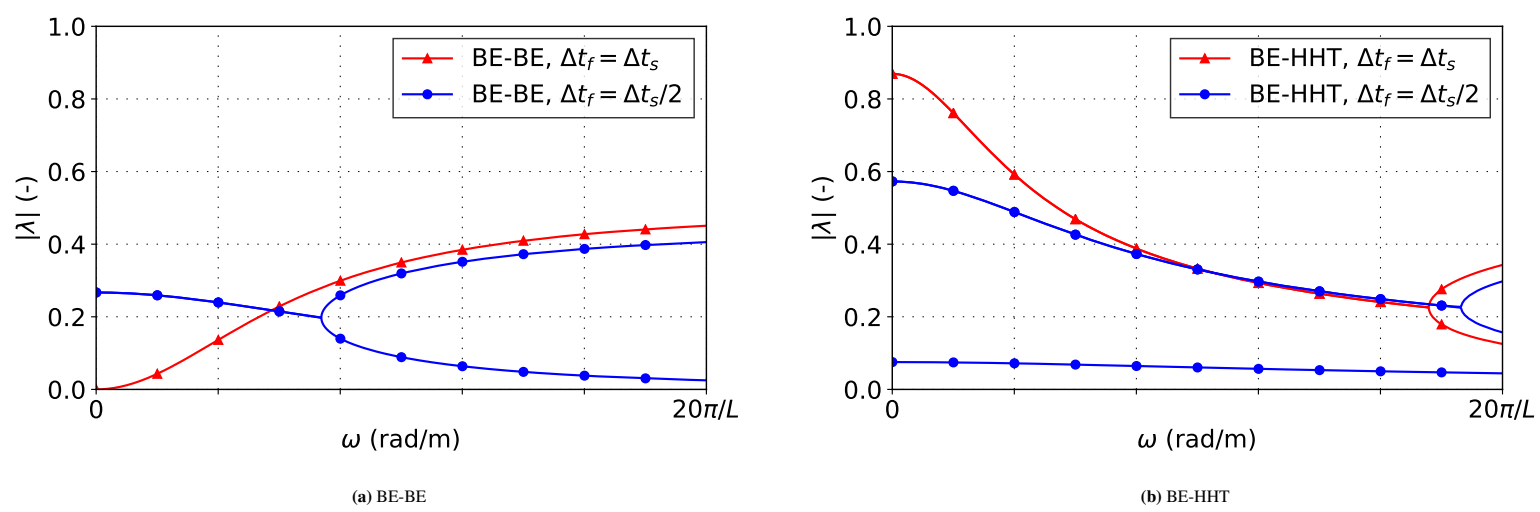

FIGURE 2 Effect of subcycling in the flow problem on the maximal amplitude of the eigenvalues corresponding to the spurious modes $(\alpha=-0.07)$.

\subsection{Subcycling in the structural problem}

\subsection{1 $\mid N_{S / F}=1 / 2$}

The analysis is repeated for a case in which $\Delta t_{s}$ is chosen equal to half of the flow time step size $\Delta t_{f}$, i.e. $N_{S / F}=1 / 2$. As opposed to Section 2.5. $\Delta t_{f}=\Delta t$ is now called 'a time step' and the two structural sub-time steps $\Delta t_{s}=\Delta t / 2$ are called 'subcycles'. Accordingly, three additional variables should be introduced $r^{n+1 / 2}, \dot{r}^{n+1 / 2}$ and $\ddot{r}^{n+1 / 2}$, representing the inner radius, the wall velocity and the acceleration at the intermediate time $n+1 / 2$ between time step $n$ and $n+1$. During each subcycle, the structural deformation is calculated for a given pressure on the fluid-structure interface. Since two subcycles are solved in each time step, one has to decide how to interpolate the wall pressure which was calculated for the entire time step. Two approaches are chosen in this paper: a pressure jump and a linearly ramped pressure profile. For the pressure jump, the pressure applied in both subcycles is equal to $p^{n+1}$ ('jump'). For a linearly ramped approach, the pressure applied during the first subcycle of time step $n+1$ is given by $\left(p^{n+1}+p^{n}\right) / 2$ and the pressure applied during the second subcycle is $p^{n+1}$ ('ramp'). Higher order pressure interpolation 
schemes are not considered as the zeroth and first order interpolation already guarantee continuous structure displacements. The reason for this is that the interface displacement is the result of a double time integration procedure, since the Neumann boundary condition defines the acceleration at the interface. A continuous interface displacement, velocity and acceleration are therefore guaranteed in case the pressure is linearly interpolated in the subcycles.

For the BE-BE combination, Equation (5) completely describes the flow problem. Equation (6) for the structure is reformulated for both subcycles (see Appendix $B$ ). The system of equations is subsequently written in the following matrix form:

$$
\begin{gathered}
A y=B z \\
y=\left[r^{n+1} \dot{r}^{n+1} \ddot{r}^{n+1} r^{n+1 / 2} \dot{r}^{n+1 / 2} \ddot{r}^{n+1 / 2} u^{n+1} p^{n+1}\right]^{T} \\
z=\left[r^{n} \dot{r}^{n} \ddot{r}^{n} r^{n-1 / 2} \dot{r}^{n-1 / 2} \ddot{r}^{n-1 / 2} u^{n} p^{n}\right]^{T} .
\end{gathered}
$$

For the BE-HHT combination, Equation (7) for the structure is transformed to accommodate for the subcycling procedure (see Appendix B).

The variables $r^{n-1 / 2}, \dot{r}^{n-1 / 2}$ and $\ddot{r}^{n-1 / 2}$ do not occur in any equation, but are added to obtain a square amplification matrix $A^{-1} B$. As a result, the matrix B contains some zero-columns. The grid size and time step size are chosen sufficiently small ( $\Delta x=10^{-4} \mathrm{~m}, \Delta t=10^{-5} \mathrm{~s}$ ), so that the eigenvalues of the amplification matrix $A^{-1} B$ are unaffected by numerical dissipation in the considered wave number region. For both discretization schemes, two eigenvalues $\lambda_{1,2}$ with an absolute value equal to one are found. Their eigenvectors (which are the same for both schemes) correspond to the solution of the one-dimensional case. The stability of the FSI problem is thus determined by the absolute value of the other six eigenvalues.

BE-BE combination In case the BE scheme is used for both the flow and solid problem in combination with the pressure ramp approach, the variable $\ddot{r}^{n}$ is also absent in the equations. Consequently, five eigenvalues corresponding to the eigenvectors $e_{3}, e_{4}, e_{5}$ and $e_{6}$, are equal to zero. These eigenvectors correspond to the zero-columns in $\mathrm{B}$; therefore, the geometric multiplicity of the eigenvalue zero equals four. When a pressure jump instead of a linear pressure profile is applied in the subcycle, neither $\ddot{r}^{n}$ nor $p^{n}$ appear in the system of equations and the five eigenvalues equal to zero correspond to the eigenvectors $e_{3}, e_{4}, e_{5}$ and $e_{6}$ and $e_{8}$ (the geometric multiplicity is equal to five in this case). The remaining eigenvalue determines the temporal stability of the system and is shown in Figure $3 \mathrm{a}$ as a function of the wave number for both pressure interpolation profiles. For $\omega$ close to zero, there will be almost no damping of the spurious modes in case of the pressure ramp approach. The pressure jump approach will slow down the convergence of the structural problem, but the entire system will be more stable compared to the ramped pressure profile. The main conclusion, however, is that the BE-BE combination remains stable regardless of the wall pressure interpolation.

BE-HHT combination Using the HHT operator to discretize the structural equation combined with the BE scheme for the flow equations, three eigenvalues $\lambda_{3,4,5}$ equal to zero are obtained, corresponding to the eigenvectors $e_{4}, e_{5}$ and $e_{6}$. Another eigenvalue is close to, but not equal to zero. These conclusions are valid for both pressure interpolation profiles. The amplitude of the two remaining eigenvalues $\lambda_{7,8}$ is shown in Figure $3 b$ for both the pressure ramp and the pressure jump approach. For small wave numbers, the HHT operator becomes unstable $(|\lambda|>1)$. As for the BE-BE scheme, the maximum amplitude of the eigenvalues is smaller for the pressure jump approach. However, the simulation using the HHT operator for the structure is unstable for both pressure interpolation schemes and cannot be used.

\subsection{2 $\mid N_{S / F}=1 / 3$}

In contrast to subcycling in the flow problem, the BE-BE scheme is only marginally stable for the lowest wavenumbers in case of $N_{S / F}=1 / 2$. It is therefore expected that the system becomes unstable for lower values of $N_{S / F}$. In order to verify this, the same study is performed for $\Delta t_{s}=\Delta t_{f} / 3$, where the pressures applied during the subcycles are $\frac{2}{3} p^{n}+\frac{1}{3} p^{n+1}, \frac{1}{3} p^{n}+\frac{2}{3} p^{n+1}$ and $p^{n+1}$, respectively. In other words, the wall pressure is interpolated linearly in between two subsequent time steps.

For the BE-BE combination, Equation (5) and the reformulated Equation (6) completely determine the system of equations (analogous to $N_{S / F}=1 / 2$ ), written in the following matrix form:

$$
\begin{gathered}
A y=B z \\
y=\left[r^{n+1} \dot{r}^{n+1} \ddot{r}^{n+1} r^{n+2 / 3} \dot{r}^{n+2 / 3} \ddot{r}^{n+2 / 3} r^{n+1 / 3} \dot{r}^{n+1 / 3} \ddot{r}^{n+1 / 3} u^{n+1} p^{n+1}\right]^{T} \\
z=\left[r^{n} \dot{r}^{n} \ddot{r}^{n} r^{n-1 / 3} \dot{r}^{n-1 / 3} \ddot{r}^{n-1 / 3} r^{n-2 / 3} \dot{r}^{n-2 / 3} \ddot{r}^{n-2 / 3} u^{n} p^{n}\right]^{T} .
\end{gathered}
$$



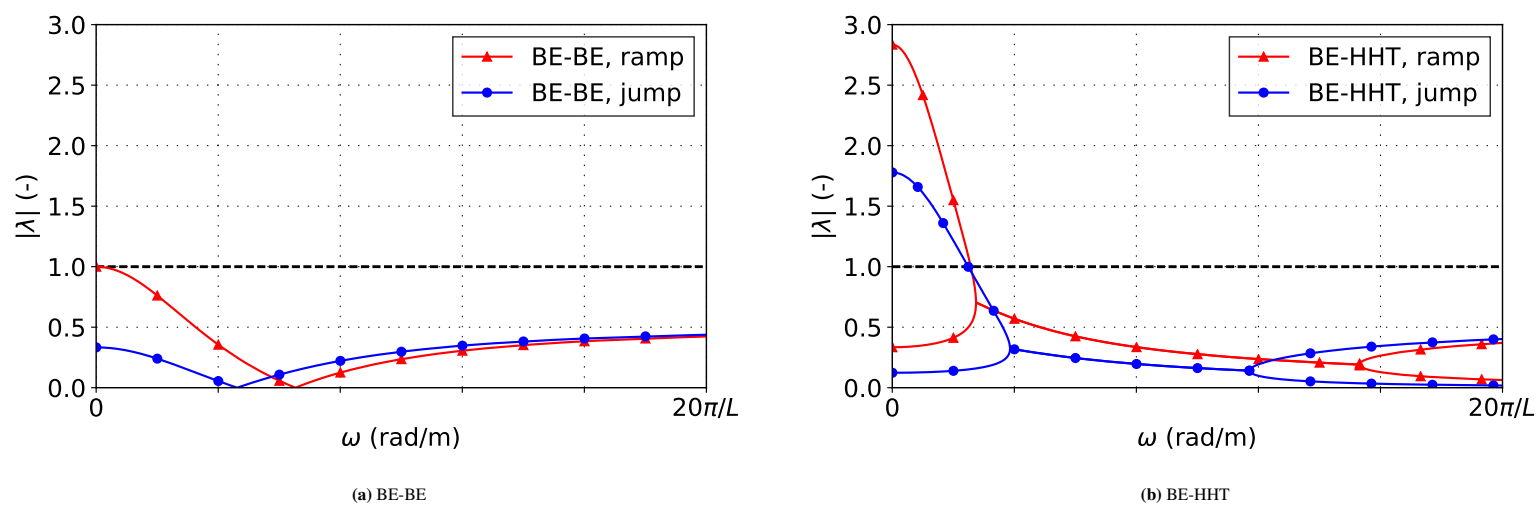

FIGURE 3 Influence of the pressure evolution (linear ramp vs. jump) during a time step on the maximal amplitude of the eigenvalues corresponding to the spurious modes $\left(\Delta t_{s}=\Delta t_{f} / 2, \alpha=-0.07\right)$.

For the BE-HHT combination, Equation (7) is transformed to accommodate for the subcycling procedure instead of Equation (6). As expected, two eigenvalues $\lambda_{1,2}$ are equal to one and six eigenvalues $\lambda_{3,4,5,6,7,8}$ are equal to zero for both discretization schemes. The eigenvectors corresponding to $\lambda_{3,4,5,6,7,8}$ are $e_{4}, e_{5}, e_{6}, e_{7}, e_{8}$ and $e_{9}$, because the variables $r^{n-1 / 3}, \dot{r}^{n-1 / 3}$, $\ddot{r}^{n-1 / 3}, r^{n-2 / 3}, \dot{r}^{n-2 / 3}$ and $\ddot{r}^{n-2 / 3}$ do not appear in the discretized equation (so, matrix B contains six zero columns).

BE-BE combination In case the BE scheme is used in both solvers, the variable $\ddot{r}^{n}$ is not present in the system of equations. This yields a seventh eigenvalue which is equal to zero, corresponding to eigenvector $e_{3}$. The two remaining eigenvalues $\lambda_{10,11}$ are compared to the eigenvalue obtained for $\Delta t_{s}=\Delta t_{f} / 2$ in Figure 4a. According to this figure, an increase in the number of subcycles has a negative effect on the stability as it causes the simulations using the BE-BE combination to become unstable for small $\omega$.

BE-HHT combination For the BE-HHT combination, only six eigenvalues are equal to zero. However, another eigenvalue $\lambda_{9}$ is close to zero and is therefore discarded in the following analysis. The two remaining eigenvalues $\lambda_{10,11}$ are shown in Figure $4 \mathrm{~b}$ and compared to the case for which $\Delta t_{s}=\Delta t_{f} / 2$. Decreasing $N_{S / F}$ causes the instability of the simulations to grow even faster. The depicted eigenvalues are calculated for $\alpha=-0.07$, but the same conclusion is found for any other value of $\alpha$.
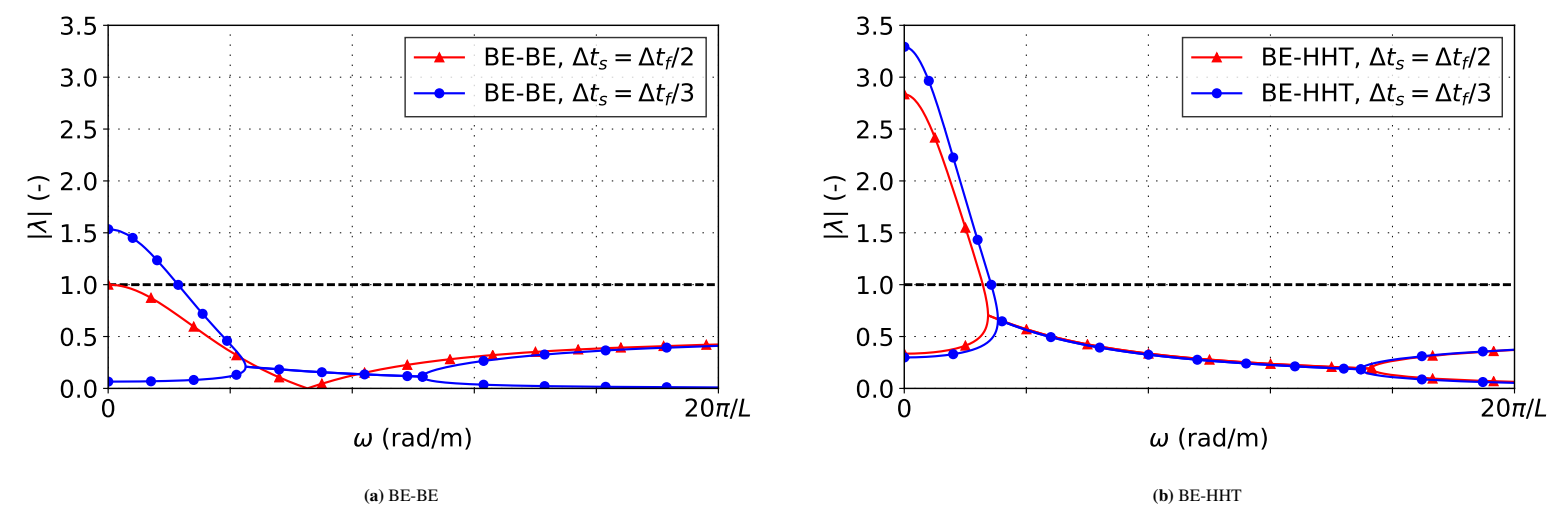

FIGURE 4 Effect of subcycling in the structural problem on the maximal amplitude of the eigenvalues corresponding to the spurious modes $(\alpha=-0.07)$. The wall pressure profile is linearly interpolated in the subcycles ('ramp'). 


\section{3 | NUMERICAL STUDY}

To verify the analytic results, a numerical study is performed in which the propagation of a sinusoidal flow wave in a straight elastic tube is simulated. The problem is solved using a nonlinear two-dimensional axisymmetric FSI model. This simplification does not introduce any loss of accuracy since gravity has been neglected. A schematic of the FSI problem is given in Figure 5 .

\section{1 | Method}

The FSI problem is solved in a partitioned way, so separate black-box solvers are used for the flow and structural computations. The flow equations are solved in an Arbitrary Lagrangian-Eulerian (ALE) formulation on a deforming mesh with the commercial code Fluent ${ }^{\circledR}$ 12.1, Ansys Inc. The displacement of the fluid grid is calculated by iteratively solving a system of linear springs between the grid nodes. The grid contains $10^{4}$ cells and the initial (undeformed) mesh is shown in Figure 6 . The parameters listed in Table 1 are used as approximate values for the flow in a tube. The fluid is modeled as an incompressible, Newtonian fluid with a viscosity of $3 \mathrm{mPas}$. At the inlet, an axial fluid velocity of $u_{0}+\frac{u_{0}}{2} \sin (2 \pi t) H(t)$ is imposed, where $H$ represents the Heaviside function (as shown in Figure 5p. Given an initial velocity field of $u_{0}=0.1 \mathrm{~m} / \mathrm{s}$, the inlet velocity has a discontinuity in its derivative at $t=0 \mathrm{~s}$. At this time, spurious modes can enter the solution and the stability and damping behaviour can be evaluated. The pressure at the outlet of the tube is set to the ambient pressure.

The structural equations are solved in a Lagrangian frame with the commercial code Abaqus ${ }^{\circledR}$ 6.7, Dassault Systèmes. The first-order upwind scheme is used to discretize the convective terms in the momentum equation. The BE scheme is selected for the temporal discretization of the flow equations. For the time integration of the structural problem, either BE or HHT is applied. Along the tube, longitudinal and circumferential wall displacements are prevented. A structured grid of 2500 quadratic elements is constructed in the structural domain. This allows the high wave numbers to be sufficiently spatially resolved. The initial structural mesh is shown in Figure 7 .

The coupling between both solvers at the interface is done through a Dirichlet-Neumann boundary condition, meaning that the pressure and shear stress are interpolated from the flow solver to the structural solver and the interface displacement is

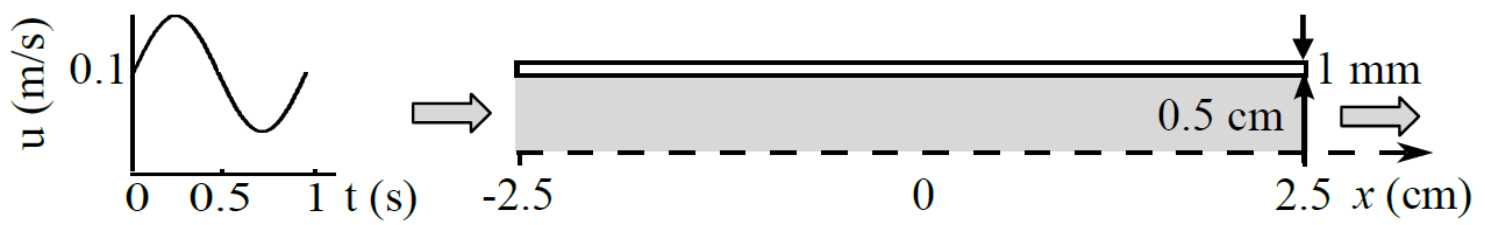

FIGURE 5 (Left) Inlet flow profile as a function of time. (Right) Schematic view of the flow/structural problem.

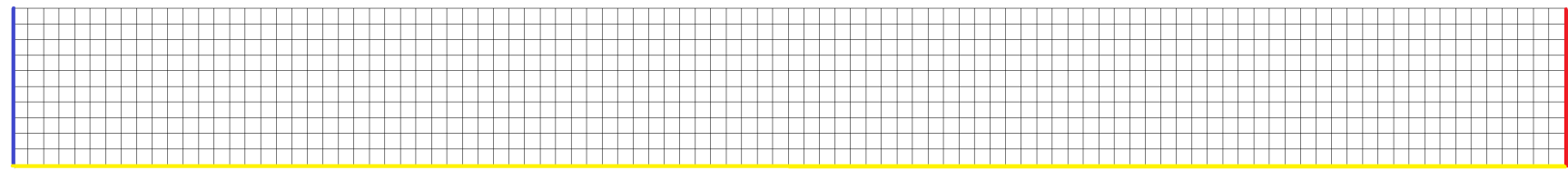

FIGURE 6 View of the undeformed ALE grid used in the flow solver. The radial dimension is subdivided in 10 elements between the symmetry axis and the tube wall. The inlet is shown in blue, the outlet in red. The symmetry axis is shown in yellow.

FIGURE 7 View of the undeformed Lagrangian grid used in the structural solver. The tube wall contains 5 element layers. The lateral and axial dimensions are not drawn to scale. 
interpolated from the structural solver to the flow solver. The equilibrium at the fluid-structure interface is established using an interface quasi-Newton algorithm with an approximation for the inverse of the Jacobian from a least-squares model (IQN-ILS) 28 . This method solves the FSI-problem with quasi-Newton iterations. As the Jacobian of the FSI-system is unknown for black-box solvers, an approximation of this Jacobian is necessary. An approximation of the inverse of the Jacobian is constructed, based on the residuals and displacements of previous coupling iterations. The IQN-ILS procedure is exactly the same as for the case without subcycling, except that more than 1 timestep is calculated either in the flow or structural solver before the next coupling iteration occurs. In the case of subcycling on the fluid side, the wrapper around the flow solver receives the data for $t^{n+1}$ from the coupling code and gives the flow solver the command to perform $N_{S / F}$ time steps, while providing boundary displacements that are interpolated in time for each of those intermediate time steps. At the beginning of the next coupling iteration, the file from time step $t^{n}$ is read in from the storage. By contrast, the subcycling in the structural solver is handled by so-called increments in each time step, with temporal interpolation in a user subroutine. The convergence criterion in the Euclidean norm for the residual of the interface displacement is set to $10^{-8} \mathrm{~m}$ and for the residual of the interface load to $10^{-2} \mathrm{~Pa}$. More severe convergence criteria have no significant impact on the results. The maximum number of coupling iterations per time step is set to 50, but typically the convergence criterion is reached within 10 coupling iterations. Accordingly, the coupling iterations have completely converged. The solution stability discussed below is therefore not related to the partitioned FSI method (which is stable), but it is the temporal stability of the discretization schemes used in both structural and flow domain when subcycling is applied. As such, the conclusions drawn from the monolithic analytic study in Section 2 are directly transposable to the case investigated here (since a monolithic solver with the same convergence criteria would yield the same solution as the applied partitioned solver). A time step size $\Delta t$ of $10^{-4} \mathrm{~s}$ is used. As the numerical behaviour of the FSI simulation is of interest, rather than the propagation of the flow wave itself, only the first 100 time steps are simulated.

\subsection{Subcycling in the flow problem}

The stability of the FSI simulation in case of subcycling in the flow solver is considered here for two cases in particular: $N_{S / F}=2$ and $N_{S / F}=10$. The coupling iterations are drawn schematically for $N_{S / F}=2$ in Figure 8 each coupling iteration contains one time step in the structure solver and two subcycles in the flow solver. A stable solution is obtained for both combinations of time integration schemes for a flow time step size $\Delta t_{f}=\Delta t_{s} / 2$ and a cubic wall displacement throughout the subcycles. This is in agreement with the results from the stability analysis shown in Figure 2 Figure 9 depicts the inlet pressure evolution throughout the subcycles during the first 15 time steps for a simulation using 2 (red curve) and 10 subcycles (blue curve), respectively. During the first subcycle of each time step a peak in inlet pressure can be observed. For the BE-BE combination this peak decreases each time step, resulting in a proper calculation of the inlet pressure after a few time steps. On the other hand, this peak continues to exist for the BE-HHT combination, leading to a discrepancy in pressure compared to the solution obtained with matching time steps.

\section{3 | Subcycling in the structural problem}

In this section, the effect of subcycling on the stability of the FSI simulation is evaluated in case $N_{S / F}<1$. The coupling scheme is schematically shown in Figure 10 The predicted displacement at time instant $n+1$ is interpolated in the subcycles, either with a discontinuous pressure increase ('jump') or with a linear variation ('ramp'), similar to what was done in the analytic study. Per coupling iteration, one time step of the flow problem is calculated while two subcycles are computed in the structure solver. In Figure 11, the inlet pressure, obtained using a simulation with a linear pressure variation throughout a time step ('ramp'), is compared to the inlet pressure of a simulation with a sudden pressure increase during the first subcycle ('jump') - both using the HHT operator. The inlet pressure, obtained using the BE scheme for both the flow and the structure and a single time step, is shown as a reference. As predicted by the analytic study, both BE-HHT simulations are unstable and the instability grows faster in case a linear pressure evolution is used.

In Figure 12 the inlet pressure evolution is shown for both combinations of time integration schemes and different values of $N_{S / F}$. The results are obtained for a linear pressure evolution throughout a time step ('ramp'). The results confirm the conclusions of the stability analysis. In case $N_{S / F}=\frac{1}{k}$, with $k$ a natural number, the BE-HHT combination is unstable for $N_{S / F} \leq \frac{1}{2}$ and the BE-BE combination for $N_{S / F} \leq \frac{1}{3}$. Values of $N_{S / F}$ down to $10^{-2}$ are simulated (not shown in Figure 12 . The smaller this time step size ratio is chosen, the faster the instability grows. 


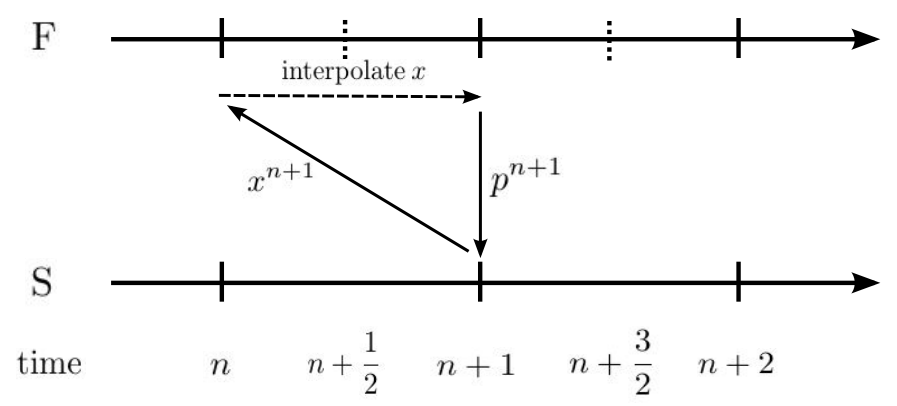

FIGURE 8 Schematic representation of the coupling iterations in the strongly-coupled FSI problem, in case $N_{S / F}=2$. The displacement and the pressure of the fluid-structure interface are represented by $x$ and $p$, respectively. The superscript denotes the time step. The transient structure (S) and flow (F) problem are depicted as separate timelines, whereas the full lines indicate the communication between the solvers. The dotted lines show other operations not related to communication between the solvers. The 'interpolate' procedure denotes the distribution of the interface displacement over the subcycles.

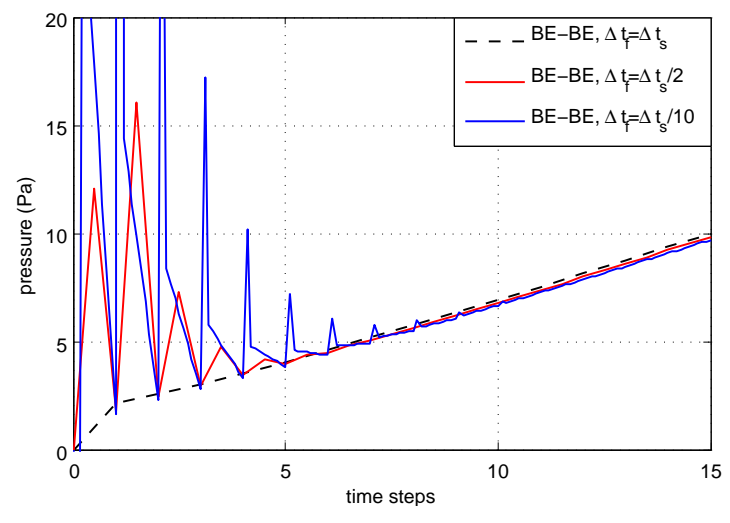

(a)

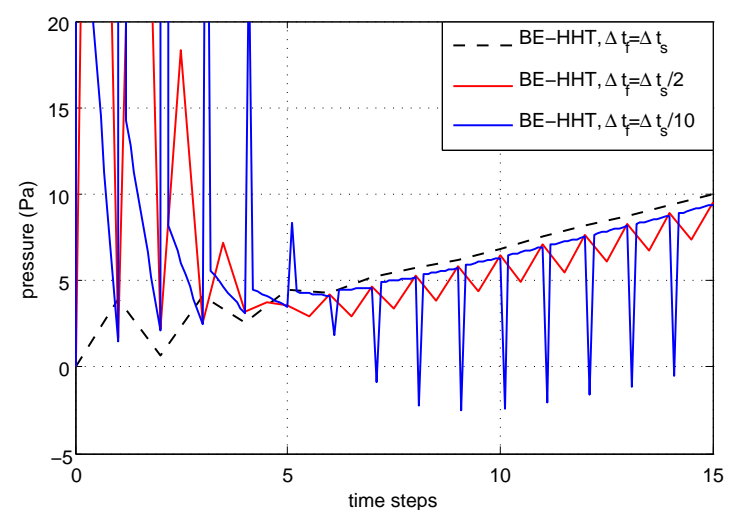

(b)

FIGURE 9 Effect of subcycling in the flow problem on the evolution of the inlet pressure using the BE-BE combination (a) and the BE-HHT combination (b) $\left(\Delta t_{s}=10^{-4} \mathrm{~s}\right)$.

\section{4 | CONCLUSIONS}

The temporal stability of the FSI problem in which the flow in a flexible tube is computed, is analyzed using different time step sizes for the fluid and the structure. A monolithic analytic study is proposed to investigate the influence of both the time discretization scheme and the subcycling ratio on the temporal stability of the solution. Subsequently, a partitioned implicit coupling scheme is used to calculate a nonlinear two-dimensional axisymmetric FSI model. To the best of the authors' knowledge, it is the first time that the effect of subcycling on temporal stability is investigated for other than explicit coupling.

For the given parameter values, the analysis yields that both BE-BE and BE-HHT combinations are stable when a smaller time step is used for the fluid than for the structure. If, on the other hand, the smallest required time step size is determined by the structural subproblem, the possibility to perform subcycling is limited: the BE-HHT combination is unstable for $N_{S / F} \leq \frac{1}{2}$ and the BE-BE combination for $N_{S / F} \leq \frac{1}{3}$. These conclusions were confirmed by the partitioned implicit FSI simulations. 


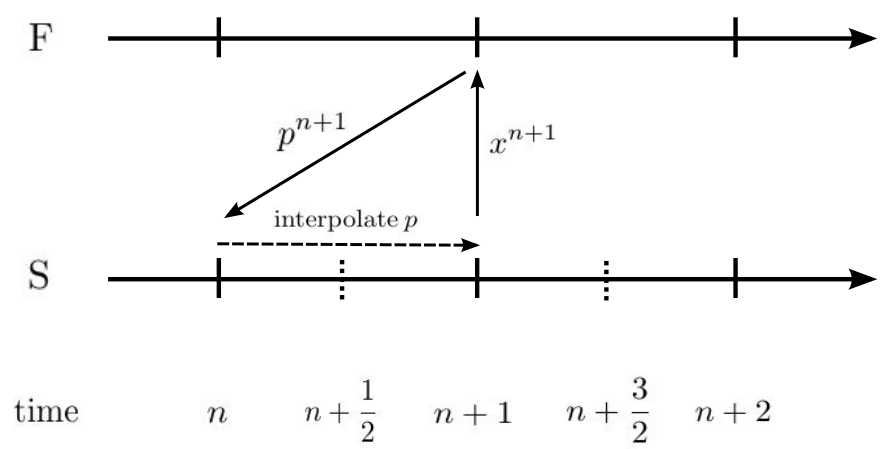

FIGURE 10 Schematic representation of the coupling iterations in the strongly-coupled FSI problem, in case $N_{S / F}=1 / 2$. The displacement and the pressure of the fluid-structure interface are represented by $x$ and $p$, respectively. The superscript denotes the time step. The transient structure (S) and flow (F) problem are depicted as separate timelines, whereas the full lines indicate the communication between the solvers. The dotted lines show other operations not related to communication between the solvers. The 'interpolate' procedure denotes the distribution of the interface pressure variation over the subcycles.

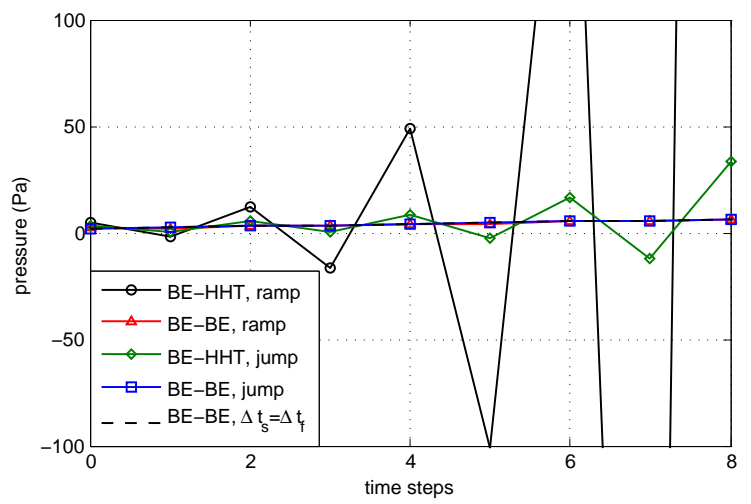

FIGURE 11 Influence of the pressure evolution (linear ramp vs. jump) during a time step on the inlet pressure evolution $\left(N_{S / F}=1 / 2\right)$.

\section{5 | ACKNOWLEDGMENTS}

The authors gratefully acknowledge the funding by the Research Foundation-Flanders (FWO), through the Ph.D. fellowship of Laurent De Moerloose. This research was also funded directly by FWO (project nr 3G008509).

\section{References}

1. De Ridder J., Degroote J., Van Tichelen K., Schuurmans P., Vierendeels J. Predicting turbulence-induced vibration in axial annular flow by means of large-eddy simulations. Journal of Fluids and Structures. 2016;61:115-131.

2. Farhat C., van der Zee K., Geuzaine P. Provably second-order time-accurate loosely-coupled solution algorithms for transient nonlinear computational aeroelasticity. Computer Methods in Applied Mechanics and Engineering. 2006;195:1973-2001.

3. Wilcox K., Paduano J., Peraire J. Low order aerodynamic models for aeroelastic control of turbomachines. In: Proceedings of 40th AIAA/ASME/ASCE/AHS/ASC structures, structural dynamics and materials (SDM) Conference:1-11; 1999. 


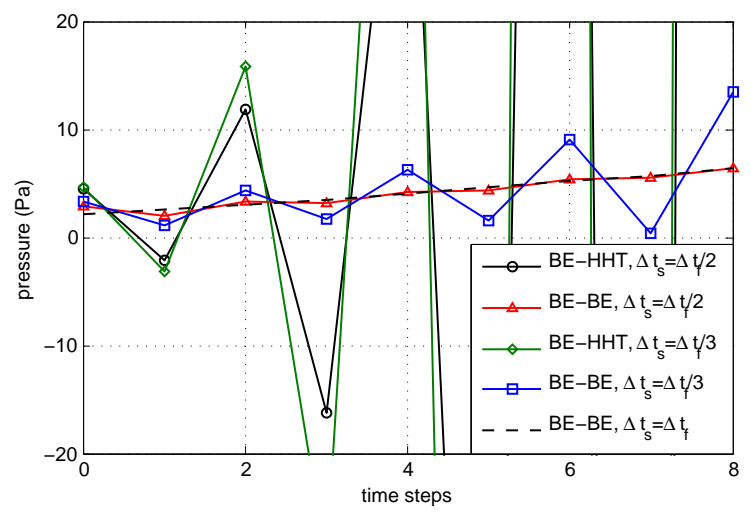

FIGURE 12 Effect of subcycling in the structural problem on the evolution of the inlet pressure $\left(\Delta t_{f}=10^{-4} \mathrm{~s}\right)$. The wall pressure profile is linearly interpolated in the subcycles ('ramp').

4. Breuer M., De Nayer G., Muensch M., Gallinger T., Wuechner R. Fluid-structure interaction using a partitioned semiimplicit predictor-corrector coupling scheme for the application of large-eddy simulation. Journal of Fluids and Structures. 2012;29:107-130.

5. Versteeg H.K., Malalasekera W. An Introduction to Computational Fluid Dynamics. Pearson Education Limited; 2007.

6. Michler C., van Brummelen E.H., Hulshoff S.J., de Borst R. The relevance of conservation for stability and accuracy of numerical methods for fluid-structure interaction. Computer Methods in Applied Mechanics and Engineering. 2003;192:4195-4215.

7. Denner F., van Wachem B. Numerical time-step restrictions as a result of capillary waves. Journal of Computational Physics. 2015;285:24-40.

8. Aksenov A., Korenev D., Shyshaeva A., Vucinic D., Mravak Z. Drop-test FSI simulation with Abaqus and FlowVision based on the direct 2-way coupling approach. In: ABAQUS Users' Conference; 2008.

9. Radtke L., Lampe T., Abdel-Maksoud M., Duester A. A partitioned simulation approach for fluid-structure interaction induced vibrations of ship propellers. In: Proceedings of the 6th European Conference on Computational Mechanics and 7th European Conference on Computational Fluid Dynamics; 2018.

10. Farhat C., Lesoinne M., Stern P. High performance solution of three-dimensional nonlinear aeroelastic problems via parallel partitioned algorithms: methodology and preliminary results. Advances in Engineering Software. 1997;28:43-61.

11. Piperno S. Explicit/implicit fluid/structure staggered procedures with a structural predictor and fluid subcycling for 2D inviscid aeroelastic simulations. International Journal for Numerical Methods in Fluids. 1997;25:1207-1226.

12. Piperno S., Farhat C., Larrouturou B. Partitioned procedures for the transient solution of coupled aeroelastic problem. Computer Methods in Applied Mechanics and Engineering. 1995;124:79-112.

13. Braun A.L., Awruch A.M. A partitioned model for fluid-structure interaction problems using hexahedral finite elements with one-point quadrature. International Journal for Numerical Methods in Engineering. 2009;79:505-549.

14. Owen D.R.J, Leonard C.R., Feng Y.T. An efficient framework for fluid-structure interaction using the lattice Boltzmann methods and immersed moving boundaries. International Journal for Numerical Methods in Engineering. 2011;87:66-95.

15. Daniel W. A study of the stability of subcycling algorithms in structural dynamics. Computer Methods in Applied Mechanics and Engineering. 1998;156:1-13.

16. Beneš M. Convergence and stability analysis of heterogeneous time step coupling schemes for parabolic problems. Applied Numerical Mathematics. 2017;121:198-222. 
17. Connors J., Howell J. A Fluid-Fluid Interaction Method Using Decoupled Subproblems and Differing Time Steps. Numerical Methods for Partial Differential Equations. 2011;28:1283-1308.

18. Newman C., Womeldorff G., Knoll D., Chacón L. A communication-avoiding implicit-explicit method for a free-surface ocean model. Journal of Computational Physics. 2016;305:877-894.

19. Zhang Q., Cen S. Multiphysics Modeling: Numerical Methods and Engineering Methods. Tshingua University Press Limited and Elsevier Inc.; 2016.

20. Causin P., Gerbeau J. F., Nobile F. Added-mass effect in the design of partitioned algorithms for fluid-structure problems. Computer Methods in Applied Mechanics and Engineering. 2005;194:4506-4527.

21. De Ridder J., Degroote J., Van Tichelen K., Vierendeels J. Predicting modal characteristics of a cluster of cylinders in axial flow: From potential flow solutions to coupled CFD-CSM calculations. Journal of Fluids and Structures. 2017;74:90-110.

22. Song M.D., Lefrançois E., Rachik M. A partitioned coupling scheme extended to structures interacting with high-density fluid flows. Computers and Fluids. 2013;84:190-202.

23. Quarteroni A., Tuveri M., Venezian A. Computational vascular fluid dynamics: problems, models and methods. Computing and Visualization in Science. 2000;2:163-197.

24. Perktold K., Rappitsch G. Mathematical modelling of local arterial flow and vessel mechanics. In: Computation Methods for Fluid Structure Interaction. Pitman Research Notes in Mathematics:230-245; 1994.

25. Degroote J., Annerel S., Vierendeels J. Stability analysis of Gauss-Seidel iterations in a partitioned simulation of fluidstructure interaction. Computers and Structures. 2010;88:263-271.

26. Hilber H.M., Hughes T.J.R., Taylor R. L. Improved numerical dissipation for time integration algorithms in structural dynamics. Earthquake Engineering and Structural Dynamics. 1977;5:283-292.

27. Newmark N.M. A method of computation for structural dynamics. ASCE Journal of the Engineering Mechanics Division. 1959;85(3):67-94.

28. Degroote J., Bathe K.J., Vierendeels J. Performance of a new partitioned procedure versus a monolithic procedure in fluidstructure interaction. Computers and Structures. 2009;87(11-12):793-801.

\section{APPENDIX}

\section{A SUBCYCLING IN THE FLOW PROBLEM}

The structural part of the problem described in the system of discretized equations $\sqrt{8 \mathrm{a}}$ ), consists of Equation $\sqrt{6}$ ) (for the BE-BE decomposition) and (7) (for the BE-HHT decomposition). The discretized equations describing the subcycled flow problem for $N_{S / F}=2$, are the following:

$$
\begin{gathered}
\frac{2 r_{0} \Delta x}{(\Delta t / 2)}\left(r^{n+1 / 2}-r^{n}\right)+\jmath r_{0}^{2} \sin (\theta) u^{n+1 / 2}+2 \jmath r_{0} u_{0} \sin (\theta) r^{n+1 / 2}+2 \delta(1-\cos (\theta)) p^{n+1 / 2}=0 \\
\frac{r_{0}^{2} \Delta x}{(\Delta t / 2)}\left(u^{n+1 / 2}-u^{n}\right)+\frac{2 r_{0} u_{0} \Delta x}{(\Delta t / 2)}\left(r^{n+1 / 2}-r^{n}\right)+(1-\exp (-\jmath \theta)+\jmath \sin (\theta)) r_{0}^{2} u_{0} u^{n+1 / 2}+2 \jmath u_{0}^{2} r_{0} \sin (\theta) r^{n+1 / 2}+J r_{0}^{2} \sin (\theta) p^{n+1 / 2}=0
\end{gathered}
$$

for the first subcycle, and

$$
\frac{2 r_{0} \Delta x}{(\Delta t / 2)}\left(r^{n+1}-r^{n+1 / 2}\right) j r_{0}^{2} \sin (\theta) u^{n+1}+2 \jmath r_{0} u_{0} \sin (\theta) r^{n+1}+2 \delta(1-\cos (\theta)) p^{n+1}=0
$$




$$
\frac{r_{0}^{2} \Delta x}{(\Delta t / 2)}\left(u^{n+1}-u^{n+1 / 2}\right)+\frac{2 r_{0} u_{0} \Delta x}{(\Delta t / 2)}\left(r^{n+1}-r^{n+1 / 2}\right)+(1-\exp (-\jmath \theta)+\jmath \sin (\theta)) r_{0}^{2} u_{0} u^{n+1}+2 \jmath u_{0}^{2} r_{0} \sin (\theta) r^{n+1}+\jmath r_{0}^{2} \sin (\theta) p^{n+1}=0
$$

for the second subcycle.

Finally, the interface displacement has to be interpolated in the flow subcycles. As discussed in section 2.5. the wall displacement in the first subcycle is given by:

$$
r^{n+1 / 2}=\frac{r^{n+1}}{2}+\frac{r^{n}}{2}-\frac{3 \ddot{r}^{n+1} \Delta t^{2}}{16}+\frac{\ddot{r}^{n} \Delta t^{2}}{16}
$$

\section{B SUBCYCLING IN THE STRUCTURAL PROBLEM}

The flow part of the problem described in the system of discretized equations $9 \mathrm{a}$ ) and (10a), consists of Equation (5). The discretized equations describing the subcycled structural problem $\left(N_{S / F}=1 / 2\right)$ are given in the following sections.

\section{B.1 BE-BE scheme}

The following equations are valid in case the linear pressure interpolation ('ramp') was used. In the first subcycle, one obtains

$$
\rho_{s} h \dot{r}^{n+1 / 2}+\left(\frac{4 \chi}{\Delta x^{4}}(1-\cos (\theta))^{2}+\frac{2 \psi}{\Delta x^{2}}(1-\cos (\theta))+\eta\right) r^{n+1 / 2}=\rho_{f} \frac{p^{n}+p^{n+1}}{2}
$$

in which the acceleration and velocity are calculated as

$$
\begin{aligned}
& \ddot{r}^{n+1 / 2}=\frac{1}{(\Delta t / 2)}\left(\dot{r}^{n+1 / 2}-\dot{r}^{n}\right) \\
& \dot{r}^{n+1 / 2}=\frac{1}{(\Delta t / 2)}\left(r^{n+1 / 2}-r^{n}\right),
\end{aligned}
$$

and in the second subcycle, the equations become

$$
\rho_{s} h \ddot{r}^{n+1}+\left(\frac{4 \chi}{\Delta x^{4}}(1-\cos (\theta))^{2}+\frac{2 \psi}{\Delta x^{2}}(1-\cos (\theta))+\eta\right) r^{n+1}=\rho_{f} p^{n+1}
$$

in which the acceleration and velocity are calculated as

$$
\begin{aligned}
& \ddot{r}^{n+1}=\frac{1}{(\Delta t / 2)}\left(\dot{r}^{n+1}-\dot{r}^{n+1 / 2}\right) \\
& \dot{r}^{n+1}=\frac{1}{(\Delta t / 2)}\left(r^{n+1}-r^{n+1 / 2}\right) .
\end{aligned}
$$

In case of the stepwise pressure interpolation ('jump'), the term $\frac{p^{n}+p^{n+1}}{2}$ in Equation B.1a has to be replaced by $p^{n+1}$.

\section{B.2 BE-HHT scheme}

The following equations are valid in case the linear pressure interpolation ('ramp') was used. In the first subcycle, the following equations are valid:

$$
\begin{aligned}
\rho_{s} h \ddot{r}^{n+1 / 2}+(1+\alpha)\left[\left(\frac{4 \chi}{\Delta x^{4}}(1-\cos (\theta))^{2}+\frac{2 \psi}{\Delta x^{2}}\right.\right. & \left.(1-\cos (\theta))+\eta) r^{n+1 / 2}-\rho_{f} \frac{p^{n}+p^{n+1}}{2}\right] \\
-\alpha & {\left[\left(\frac{4 \chi}{\Delta x^{4}}(1-\cos (\theta))^{2}+\frac{2 \psi}{\Delta x^{2}}(1-\cos (\theta))+\eta\right) r^{n}-\rho_{f} p^{n}\right]=0 . }
\end{aligned}
$$

The operator definition is completed by the Newmark formula for acceleration and velocity integration:

$$
\begin{aligned}
\ddot{r}^{n+1 / 2} & =\frac{1}{\beta\left(\Delta t^{2} / 4\right)}\left(r^{n+1 / 2}-r^{n}\right)-\frac{1}{\beta(\Delta t / 2)} \dot{r}^{n}-\left(\frac{1}{2 \beta}-1\right) \ddot{r}^{n} \\
\dot{r}^{n+1 / 2} & =\dot{r}^{n}+\frac{\Delta t}{2}(1-\gamma) \ddot{r}^{n}+\frac{\Delta t}{2} \gamma \ddot{r}^{n+1 / 2} .
\end{aligned}
$$


For the second subcycle, one obtains

$$
\begin{aligned}
\rho_{s} h \ddot{r}^{n+1}+(1+\alpha)\left[\left(\frac{4 \chi}{\Delta x^{4}}(1-\cos (\theta))^{2}\right.\right. & \left.\left.+\frac{2 \psi}{\Delta x^{2}}(1-\cos (\theta))+\eta\right) r^{n+1}-\rho_{f} p^{n+1}\right] \\
& -\alpha\left[\left(\frac{4 \chi}{\Delta x^{4}}(1-\cos (\theta))^{2}+\frac{2 \psi}{\Delta x^{2}}(1-\cos (\theta))+\eta\right) r^{n+1 / 2}-\rho_{f} \frac{p^{n}+p^{n+1}}{2}\right]=0 \\
\ddot{r}^{n+1} & =\frac{1}{\beta\left(\Delta t^{2} / 4\right)}\left(r^{n+1}-r^{n+1 / 2}\right)-\frac{1}{\beta(\Delta t / 2)} \dot{r}^{n+1 / 2}-\left(\frac{1}{2 \beta}-1\right) \ddot{r}^{n+1 / 2} \\
\dot{r}^{n+1} & =\dot{r}^{n+1 / 2}+\frac{\Delta t}{2}(1-\gamma) \ddot{r}^{n+1 / 2}+\frac{\Delta t}{2} \gamma \ddot{r}^{n+1} .
\end{aligned}
$$

In case of the stepwise pressure interpolation ('jump'), the term $\frac{p^{n}+p^{n+1}}{2}$ in Equations B.3a and B.4a) has to be replaced by $p^{n+1}$. 